\title{
Application of Formula Method for Bound State Problems in Schrödinger Equation
}

\section{${ }^{* 1}$ EBOMWONYI, O; ${ }^{2}$ ONATE, C A; ${ }^{3}$ ODEYEMI, O E}

\author{
${ }^{l}$ Department of Physics, University of Benin, Benin City, Nigeria \\ ${ }^{2}$ Department of Physical Sciences, Landmark University, Omu-Aran, Nigeria \\ ${ }^{3}$ Department of Science Laboratory Technology, Federal College of Animal Health and Production Technology, Ibadan, Nigeria \\ *Corresponding Author Email: osarodion.ebomwonyi@ uniben.edu
}

\begin{abstract}
By using Formula method for bound state problems, we have obtained the approximate analytical solution of the radial Schrödinger equation for Varshni potential model. The energy eigenvalues and the corresponding eigenfunctions are calculated in closed forms. In the process, eigen values for $a=b=1, a=-1, b=-2$, and $b=-1, a=-2$ for $2 p, 3 p, 3 d, 4 p$ and $4 f$ states with three different values of the potential range were generated using the Varshni potential. Special cases of the potential are equally studied. Some numerical results have been presented which showed a good agreement with previous results obtained from another method.
\end{abstract}

\section{DOI: https://dx.doi.org/10.4314/jasem.v23i2.19}

Copyright: Copyright (C) 2019 Ebomwonyi et al. This is an open access article distributed under the Creative Commons Attribution License (CCL), which permits unrestricted use, distribution, and reproduction in any medium, provided the original work is properly cited.

Dates: Received: 16 December 2018; Revised: 20 January 2019; Accepted 29 January 2019

Keywords: Varshni potential, Schrödinger equation, Formula method, Bound state

The study of exactly solvable problem has attracted much attention in theoretical physics since the development of quantum mechanics. For instance, the solutions of the non-relativistic Schrödinger equation for a hydrogen atom and a harmonic oscillator in three dimensions represent two typical examples in quantum mechanics (Schiff, 1955; Landau and Lifshitz, 1977). There are few traditional techniques or methods used to solve second-order differential equation in order to obtain the solutions of the quantum systems. These methods include NikiforovUvarov method (Ebomwonyi et al., 2017; Ikot et al., 2015; Onate and Idiodi, 2015; Hamzavi and Ikhdair, 2012), asymptotic iteration method (Bayrak et al., 2007; Falaye et al., 2013; Bayrak and Boztosun, 2007; Ateser et al., 2007), supersymmetry shape invariance and solvable potential (Zarrinkamar et al., 2010; Jia et al., 2011; Hassanabadi, 2011; Onate et al., 2017), factorization method (Onyeaju et al., 2016), new exact quantization rule (Dong and Gonzalez-Cisneros, 2008; $\mathrm{Gu}$ et al., 2009) and others. Recently, Falaye et al. (2014) developed the Formula method for bound state problems from asymptotic iteration method. This method seems to be very effective, but the simplification in obtaining the energy equation is somehow technical; hence, resulting in little report on it in literature. This necessitates the present study; and in this paper, we intend to investigate the nonrelativistic Schrödinger equation with the Varshni potential function in the framework of the Formula method for bound state problems. To the best of our knowledge, this is the first time the Schrödinger equation is being studied with the Varshni potential function using the Formula method.

The Varshni potential function is given as (Varshni, 1957; Oluwadare and Oyewumi, 2017)

$V(r)=a-\frac{a b e^{-\delta r}}{r}$,

Where, $a$ and $b$ are the potential strengths, $\delta$ is the screening parameter which controls the shape of the potential energy curve and $r$ is the internuclear separation. The Varshni potential function is a short range repulsive potential energy function that plays an important role in both chemical and molecular physics (Varshni, 1957). The potential was used by Kaxiras and Pandey to describe the 2-body energy portion of multi-body condensed matter. This potential is used generally to describe bound states of the interaction of systems and has been applied in both classical and modern physics. The Varshni potential function can be adjusted to adjustable Varshni potential function as shown in Eq. (2)

$V(r)=a-\frac{v b e^{-\delta r}}{r}$,

Where $v$ is an adjusted parameter. The adjustable Varshni potential function can be transformed into 
other useful physical potential function. For instance, when the adjustable parameter $v=1$, and

$$
\lim _{a \rightarrow 0} V(r)=-\frac{b e^{-\delta r}}{r}
$$

the adjustable Varshni function turns to Yukawa potential model. Similarly, when the adjustable parameter $v=0$, the adjustable Varshni potential function becomes a constant potential

$V(r)=a$. (4)

\section{MATERIALS AND METHODS}

In this section, the bound state system was applied to calculate the energy equation and the wave function of the three dimensional radial Schrödinger equation with the Varshni potential function in Eq. (2) by employing the Formula method.

Formula Method: Given a second-order differential equation of the form

$$
\left(\frac{d^{2}}{d r^{2}}+\frac{c_{1}-c_{2} s}{\mathrm{~s}\left(1-c_{3} s\right)} \frac{d}{d s}+\frac{\xi_{1} s^{2}+\xi_{2} s+\xi_{3}}{s^{2}\left(1-c_{3} s\right)^{2}}\right) \psi(s)=0
$$

Falaye et al. (2015), gave the condition for energy equation as

$$
\alpha_{1}^{2}=\left[\frac{\alpha_{0}^{2}-\alpha_{1}^{2}-\left[\frac{1-2 n}{2}-\frac{1}{2 c_{3}}\left(c_{2}-\sqrt{\left(c_{3}-c_{2}\right)^{2}-4 \xi_{1}}\right)\right]^{2}}{2\left[\frac{1-2 n}{2}-\frac{1}{2 c_{3}}\left(c_{2}-\sqrt{\left(c_{3}-c_{2}\right)^{2}-4 \xi_{1}}\right)\right]}\right]^{2}
$$

,(6)

Where

$$
\begin{aligned}
& \alpha_{0}^{2}=\frac{\left(1-c_{1}\right)+\sqrt{\left(1-c_{1}\right)^{2}-4 \xi_{3}}}{2} \\
& \alpha_{1}^{2}=\frac{1}{2}+\frac{c_{1}}{2}-\frac{c_{2}}{2 c_{3}}+\sqrt{\left(\frac{1}{2}+\frac{c_{1}}{2}-\frac{c_{2}}{2 c_{3}}\right)^{2}-\frac{\xi_{1}}{c_{3}^{2}}-\frac{\xi_{2}}{c_{2}}-\xi_{3}},(8)
\end{aligned}
$$

and the corresponding wave function is given as

$$
\psi_{n \ell}(s)=N_{n \ell} s^{\alpha_{0}}(1-s)_{2}^{\alpha_{1}} F_{1}\left(-n, n+2 \alpha_{0}+2 \alpha_{1} ; 2 \alpha_{0}+1 ; c_{3} s\right) .(9)
$$

\section{RESULTS AND DISCUSSION}

Bound State Solutions: In this section, we calculate the energy equation and the wave function of the three dimensional radial Schrödinger equation with the Varshni potential function. The time independent
Schrödinger equation incorporating the potential function $V(r)$, nonrelativistic energy $E_{n \ell}$ is given as

$\left[\frac{d^{2}}{d r^{2}}+\frac{2 \mu}{\hbar^{2}}\left(E_{n, \ell}-V(r)-\frac{\ell(\ell+1) \hbar^{2}}{2 \mu r^{2}}\right)\right] R_{n, \ell}(r)=0$,

where $\ell$ is the angular momentum quantum number, $n$ is the orbital quantum number, $R_{n, \ell}(r)$ is the wave function, $\mu$ is the reduced mass and $\hbar$ is the reduced Planck constant. Due to the presence of the centrifugal term $\frac{1}{r^{2}}$ in Eq. (10), we cannot obtain a solution for $\ell=0$ state, therefore, we must apply an approximation scheme to deal with the centrifugal term. For short range potential, it is found that the following approximation

$\frac{1}{r^{2}} \approx \frac{\delta^{2}}{\left(1-e^{-\delta r}\right)^{2}}$

is a good approximation to the centrifugal term which is valid for $\delta \square 1$.

Now, substituting the potential in Eq. (1) and the approximation in Eq. (11) into Eq. (10) and by defining a variable of the form $y=e^{-\delta r}$, Eq. (10) becomes

$\left[\frac{d^{2}}{d y^{2}}+\frac{1-y}{y(1-y)} \frac{d}{d y}+\frac{A y^{2}+B y+C}{[y(1-y)]^{2}}\right] R_{n, \ell}(y)=0, \cdot$

Where

$$
\begin{aligned}
& A=\frac{2 \mu E_{n, \ell}}{\delta^{2} \hbar^{2}}-\frac{2 \mu a}{\delta^{2} \hbar^{2}}-\frac{2 \mu a b}{\delta \hbar^{2}},(13) \\
& B=-\frac{4 \mu E_{n, \ell}}{\delta^{2} \hbar^{2}}+\frac{4 \mu a}{\delta^{2} \hbar^{2}}+\frac{2 \mu a b}{\delta \hbar^{2}} \\
& C=\frac{2 \mu E_{n, \ell}}{\delta^{2} \hbar^{2}}-\frac{2 \mu a}{\delta^{2} \hbar^{2}}-\ell(\ell+1)
\end{aligned}
$$

Comparing Eq. (12) with Eq. (5), we deduced the following

$$
\left.\begin{array}{l}
c_{1}=c_{2}=c_{3}=1, \\
\alpha_{0}^{2}=\frac{2 \mu a}{\delta^{2} \hbar^{2}}-\frac{2 \mu E_{n, \ell}}{\delta^{2} \hbar^{2}}+\ell(\ell+1), \\
\alpha_{1}^{2}=(1+\ell)^{2} .
\end{array}\right\} .
$$

Substituting Eq. (16) into Eq. (6), we have energy equation of the Varshni potential function as 
$E_{n, \ell}=a+\frac{\delta^{2} \ell(\ell+1) \hbar^{2}}{2 \mu}-\frac{\delta^{2} \hbar^{2}}{2 \mu}\left[\frac{\frac{2 \mu a b}{\delta \hbar^{2}}-\ell(\ell+1)-(1+n+\ell)^{2}}{2(1+n+\ell)}\right]^{2} \cdot \quad \begin{aligned} & \text { The corresponding wave function is given as } \\ & R_{n \ell}(y)=N_{n \ell} y^{\sqrt{\frac{2 \mu a}{\delta^{2} \hbar^{2}}-\frac{2 \mu E_{n, \ell}}{\delta^{2} \hbar^{2}}+\ell(\ell+1)}}(1-y)^{1+\ell} \times\end{aligned}$

$$
{ }_{2} F_{1}\left(-n, n+\frac{4 \mu a}{\delta^{2} \hbar^{2}}-\frac{4 \mu E_{n, \ell}}{\delta^{2} \hbar^{2}}+2 \ell^{2}+4 \ell+2 ; \frac{4 \mu a}{\delta^{2} \hbar^{2}}-\frac{4 \mu E_{n, \ell}}{\delta^{2} \hbar^{2}}+2 \ell^{2}+2 \ell+1 ; y\right)
$$

In Table 1, we generated eigenvalues for Varshni potential with $a=b=-1, a=-1, b=-2$, and $b=-1, a=-2$ for $2 \mathrm{p}, 3 \mathrm{p}, 3 \mathrm{~d}, 4 \mathrm{p}, 4 \mathrm{~d}$ and $4 \mathrm{f}$ states with three different values of the potential range. It is observed that as the potential range increases, the energy of the system increases. Similarly, at constant value of $a$, the energy of the system decreases as $b$, the strength of the potential decreases. This same effect is observed when $a$ and $b$ is interchanged. The energy of the system is also found to be increasing as both the orbital and angular momentum quantum number increases respectively. In Table 2, we presented eigenvalues for adjusted Varshni potential and Yukawa potential for $1 \mathrm{~s}, 2 \mathrm{~s}, 2 \mathrm{p}, 3 \mathrm{~s}, 3 \mathrm{p}$ and $3 \mathrm{~d}$ states. The energy equation for these potentials were obtained in special cases. To test the accuracy of these results, we compared the result of Yukawa potential with the result of Hamzavi et al. (2013) who used parametric NikiforovUvarov method. As it can be seen from the Table 2, our results are in good agreement with the previous result. However, our results indicated that the results for adjusted Varshni potential and Yukawa potential are equivalent as considered in the special cases.

Special Cases: Using the adjustable potential in Eq. (2), the energy equation above remains the same except that the adjustable parameter will appear. When the adjustable parameter $v=1$ and $a=0$, the energy equation for the adjustable Varshni potential turns to the energy equation for the Yukawa potential as

$$
E_{n, \ell}=\frac{\delta^{2} \ell(\ell+1) \hbar^{2}}{2 \mu}-\frac{\delta^{2} \hbar^{2}}{2 \mu}\left[\frac{\frac{2 \mu b}{\delta \hbar^{2}}-\ell(\ell+1)-(1+n+\ell)^{2}}{2(1+n+\ell)}\right]^{2}
$$

When the adjustable parameter $v=0$, the energy equation for the adjusted Varshni potential becomes energy equation for constant potential as

$$
E_{n, \ell}=a+\frac{\delta^{2} \ell(\ell+1) \hbar^{2}}{2 \mu}-\frac{\delta^{2} \hbar^{2}}{2 \mu}\left[-\frac{\ell(\ell+1)}{2(1+n+\ell)}-\frac{(1+n+\ell)}{2}\right]^{2}
$$

Table 1: Bound state energy eigenvalues of the Varshni potential as a function of the screening parameter with $2 \mu=\hbar=1$.

\begin{tabular}{lllll}
\hline State & $\delta$ & $E_{n, \ell}$ & $E_{n, \ell}$, & $E_{n, \ell}$, \\
& & $a=b=-1$ & $\begin{array}{l}a=-1, \\
b=-2\end{array}$ & $a=-2$, \\
& & & $b=-1$ \\
\hline $2 p$ & 0.001 & -1.0617502 & -1.2485002 & -2.2485002 \\
& 0.050 & -1.0256250 & -1.1756250 & -2.1756250 \\
$3 p$ & 0.100 & -0.9900000 & -1.1025000 & -2.1025000 \\
& 0.001 & -1.0271680 & -1.1098902 & -2.1098902 \\
3 & 0.050 & -1.0006250 & -1.0534028 & -2.0534028 \\
& 0.100 & -0.9802778 & -1.0025000 & -2.0025000 \\
$4 p$ & 0.001 & -1.0269447 & -1.1094470 & -2.1094470 \\
& 0.050 & -0.9867361 & -1.0284028 & -2.0284028 \\
& 0.100 & -0.9469444 & -0.9469444 & -1.9469444 \\
$4 d$ & 0.001 & -1.0150656 & -1.0613781 & -2.0613781 \\
& 0.050 & -0.9951563 & -1.0139062 & -2.0139062 \\
4 & 0.100 & -0.9900000 & -0.9806250 & -1.9806250 \\
$4 f$ & 0.001 & -1.0149391 & -1.0611266 & -2.0611266 \\
& 0.050 & -0.9851563 & -0.9976563 & -1.9976563 \\
& 0.100 & -0.9625000 & -0.9406250 & -1.9406250 \\
& 0.001 & -1.0147502 & -1.0607502 & -2.0607502 \\
& 0.050 & -0.9725000 & -0.9756250 & -1.9756250 \\
& 0.100 & -0.9306250 & -0.8900000 & -1.8900000 \\
\hline
\end{tabular}


Table 2: Bound state energy eigenvalues of the adjusted Varshni and Yukawa potentials as a function of the screening parameter with $2 \mu=\hbar=1$.

\begin{tabular}{lllll}
\hline State & $\delta$ & $\begin{array}{l}E_{n, \ell}, c=3 \\
a=-0.01,\end{array}$ & $\begin{array}{l}E_{n, \ell}, c=1 \\
a=0,\end{array}$ & $\begin{array}{l}\text { Hamzavi et al. } \\
(2013)\end{array}$ \\
& & $b=1$ & $b=-3$ & \\
\hline \multirow{3}{*}{$1 s$} & 0.001 & -2.258500 & -2.248500 & -2.248500 \\
& 0.005 & -2.252500 & -2.242251 & -2.242251 \\
\multirow{3}{*}{$2 s$} & 0.010 & -2.245025 & -2.235025 & -2.235025 \\
& 0.001 & -0.571001 & -0.561001 & -0.561001 \\
& 0.005 & -0.565625 & -0.555025 & -0.555025 \\
$2 d$ & 0.010 & -0.557600 & -0.547600 & -0.547600 \\
& 0.001 & -0.570250 & -0.560250 & -0.560250 \\
3 & 0.005 & -0.561256 & -0.551256 & -0.551256 \\
& 0.010 & -0.550025 & -0.540025 & -0.540025 \\
& 0.001 & -0.258502 & -0.248502 & -0.248502 \\
$3 p$ & 0.005 & -0.252556 & -0.242556 & -0.242556 \\
& 0.010 & -0.245225 & -0.235225 & -0.235225 \\
& 0.001 & -0.258168 & -0.248168 & -0.248168 \\
$3 d$ & 0.005 & -0.250867 & -0.240867 & -0.240867 \\
& 0.010 & -0.241803 & -0.231803 & -0.231803 \\
& 0.001 & -0.257500 & -0.247500 & -0.247500 \\
& 0.005 & -0.247506 & -0.237506 & -0.237506 \\
& 0.010 & -0.235025 & -0.225025 & -0.225025 \\
\hline
\end{tabular}

Conclusion: In this study, the approximate solution of the Schrödinger equation is obtained using a suitable approximation to the centrifugal term in the framework of Formula method for bound state problem. By change of parameters, an adjusted Varshni potential model was transformed to Yukawa potential and constant potential respectively. The result obtained shows a good agreement with a previous result obtained by another method. This confirmed the accuracy of the unpopularly used Formula method for bound state problem.

\section{REFERENCES}

Ateser, E; Ciftci, H; Ugurlu, M (2007). A study of the Schrödinger equation with the linear potential by the asymptotic iteration method in 3D. Chin. J. Phys. 45, 346-351.

Bayrak, O; Boztosun, I (2007). Bound state solutions of the Hulthen potential by using the asymptotic iteration method. Phys. Scr. 76, 92-96.

Bayrak, O; Boztosun, I; Ciftci, H. (2007). Exact analytical solutions to the Kratzer potential by the asymptotic iteration method. Int. J. Quantum Chem. 107, 540-544.

Dong, S H; Gonzalez-Cisneros, A (2008). Energy spectral of the hyperbolic and Second PöschlTeller like potentials solved by new quantization rule. Ann. Phys. 323, 1136-1149.

Ebomwonyi, O; Onate, CA; Onyeaju, M C; Ikot, AN (2017). Any $l$-states solutions of the Schrödinger equation interacting with Hellmann-generalized Morse potential model. Karbala Int. J. Mod. Sci. 3, 59-68.

Falaye, B J; Ikhdair, S M; Hamzavi, M (2014). Formula method for bound state problems. FewBody Syst. 56, 63 (1-16).

Falaye, BJ; Oyewumi, KJ; Ibrahim, TT; Punyasena, M A; Onate, C.A (2013). Bound state solutions of the Manning-Rosen potential. Can. J. Phys. 91, 98104.

Gu, X Y; Dong, S H; Ma, Z Q (2009). Energy spectra for modified Rosen-Morse potential solved by the exact quantization rule. J. Phys. A: Math. Theor. 42, 035303 (1-8).

Hamzavi, M; Ikhdair, SM (2012). Any J-state solution of the Duffin-Kemmer-Petiau equation for a vector deformed Woods-Saxon potential. Few-Body Syst. $53,461-471$.

Hamzavi, M; Thylwe, KE; Rajabi, AA (2013). Approximate bound states solution of the Hellmann potential. Commun. Theor. Phys. 60, 18

Hassanabadi, H; Zarrinkamar, S; Rahimov, H (2011). Approximate solutions of D-Dimensional KleinGordon equation with Hulthen-type potential via SUSYQM. Commun. Theor. Phys.56, 423-428. 
Ikot, A N; Hassanabadi, H; Abbey, T M (2015). Spin and pseudospin symmetries of Hellmann potential with three tensor interactions using NikiforovUvarov method. Commun. Theor. Phys. 64, 637643.

Jia, C S; Guo, P; Diao, YF; Yi, LZ; Xie, XJ (2007). Solutions of Dirac equations with the Poschl-Teller potential. Eur. Phys. J. A 34, 41-48.

Landau, L D; Lifshitz, E M (1977). Quantum Mechanics, (Non-Relativistic Theory), $3^{\text {rd }}$ ed., Pergamon, New York.

Oluwadare, OJ; Oyewumi, KJ (2017). Scattering state solutions of the Duffin-Kemmer-Petiau equation with the Varshni potential model. Eur. Phys. J. A $53,29(1-6)$.

Onate, C A; Idiodi, J O A (2015). Eigensolutions of the Schrödinger equation with some physical potentials. Chin. J. Phys. 53, 120001-10

Onate, CA; Onyeaju, MC; Ikot, AN; Ebomwonyi, O (2017). Eigen solutions and entropic system for
Hellmann potential in the presence of the Schrödinger equation. Eur. Phys. J. Plus 132, 462 (1-11).

Onyeaju, M C; Idiodi, J O A; Ikot, AN; Solaimani, M; Hassanabadi, H (2016). Linear and nonlinear optical properties in spherical quantum dots: generalized Hulthen potential. Few-Body Syst. 57, 793-805.

Schiff, L I (1955). Quantum Mechanics, $3^{\text {rd }}$ ed. McGraw-Hill Book Co., New York.

Varshni, YP (1957). Comparative study of potential energy functions for diatomic molecules, Rev. Mod. Phys. 29, 664-682.

Zarrinkamar, S; Rajabi, AA; Hassanabadi, H (2010). Dirac equation for the harmonic scalar and vector potentials and linear plus Coulomb like tensor potential: the SUSY approach. Ann. Phys.325, 2522-2528. 